\title{
Three Order Parameters in Quantum XZ Spin-Oscillator Models with Gibbsian Ground States
}

\author{
Teunis C. DORLAS ${ }^{\dagger}$ and Wolodymyr I. SKRYPNIK $\ddagger$ \\ $\dagger$ Dublin Institute for Advanced Studies, School of Theoretical Physics, \\ 10 Burlington Road, Dublin 4, Ireland \\ E-mail:dorlas@stp.dias.ie \\ $\ddagger$ Institute of Mathematics of NAS of Ukraine, Kyiv, Ukraine \\ E-mail:skrypnik@imath.kiev.ua
}

Received October 29, 2007, in final form January 08, 2008; Published online January 17, 2008

Original article is available at http://www.emis.de/journals/SIGMA/2008/007/

\begin{abstract}
Quantum models on the hyper-cubic $d$-dimensional lattice of spin- $\frac{1}{2}$ particles interacting with linear oscillators are shown to have three ferromagnetic ground state order parameters. Two order parameters coincide with the magnetization in the first and third directions and the third one is a magnetization in a continuous oscillator variable. The proofs use a generalized Peierls argument and two Griffiths inequalities. The class of spinoscillator Hamiltonians considered manifest maximal ordering in their ground states. The models have relevance for hydrogen-bond ferroelectrics. The simplest of these is proven to have a unique Gibbsian ground state.
\end{abstract}

Key words: order parameters; spin-boson model; Gibbsian ground state

2000 Mathematics Subject Classification: 82B10; 82B20; 82B26

\section{Introduction}

In this paper we consider quantum lattice models of oscillators interacting with spins whose variables are indexed by the sites of a hyper-cube $\Lambda$ with the finite number of sites $|\Lambda|$ in the hyper-cubic lattice $\mathbb{Z}^{d}$. Interaction is considered to be short-range and translation invariant. The corresponding Hamiltonian $H_{\Lambda}$ is expressed in terms of the oscillators variables $q_{\Lambda}=$ $\left(q_{x}, x \in \Lambda\right) \in \mathbb{R}^{|\Lambda|}$ and spin $\frac{1}{2}$ Pauli matrices $S_{\Lambda}^{l}=\left(S_{x}^{l}, x \in \Lambda, l=1,3\right)$, defined in the tensor product of the $2^{|\Lambda|}$-dimensional Euclidean space and the space of square integrable functions $\mathbb{L}_{\Lambda}^{2}=\left(\otimes \mathbb{E}^{2}\right)^{|\Lambda|} \otimes L^{2}\left(\mathbb{R}^{|\Lambda|}\right)$,

$$
H_{\Lambda}=\sum_{x \in \Lambda}\left[-\partial_{x}^{2}+\mu^{2}\left(q_{x}+\eta \phi_{x}\left(S_{\Lambda}^{3}\right)\right)^{2}-\mu\right]+\sum_{A \subseteq \Lambda} J_{A} S_{[A]}^{1}+V_{\Lambda}, \quad \mu \geq 0, \quad \eta \in \mathbb{R}
$$

where $\partial_{x}$ is the partial derivative in $q_{x}, J_{A}$ and $V_{\Lambda}$ are real-valued measurable functions, the first of which depends on $q_{\Lambda}$ and the second on $S_{\Lambda}^{3}, q_{\Lambda}$ and the translation invariant $\phi_{x}$ is given by (see Remark 2 in the end of the paper)

$$
\phi_{x}\left(s_{\Lambda}\right)=\sum_{A \subseteq \Lambda} J_{0}(x ; A) s_{[A]}, \quad J_{0}(x ; x)=1 .
$$

${ }^{\star}$ This paper is a contribution to the Proceedings of the Seventh International Conference "Symmetry in Nonlinear Mathematical Physics" (June 24-30, 2007, Kyiv, Ukraine). The full collection is available at http://www.emis.de/journals/SIGMA/symmetry2007.html 
For products of operators, functions and variables we use the following notation: $B_{[A]}=\prod_{x \in A} B_{x}$. The scalar product in $\left(\otimes \mathbb{E}^{2}\right)^{|\Lambda|}, \mathbb{L}_{\Lambda}^{2}$ will be denoted by $(\cdot, \cdot)_{0},(\cdot, \cdot)$, respectively. The Schwartz space of test functions on $\mathbb{R}^{n}$ will be denoted by $\mathbb{S}\left(\mathbb{R}^{n}\right)$.

We require that the Hamiltonian is well defined and bounded from below on $\left(\otimes \mathbb{E}^{2}\right)^{|\Lambda|} \otimes$ $C_{0}^{\infty}\left(\mathbb{R}^{|\Lambda|}\right)$, i.e. the tensor product of the $2^{|\Lambda|}$-dimensional Euclidean space and the space of infinitely differentiable functions with compact supports. The ground state average for an observable (operator) $F$ is determined by

$$
\langle F\rangle_{\Lambda}=Z_{\Lambda}^{-1}\left(\Psi_{\Lambda}, F \Psi_{\Lambda}\right), \quad Z_{\Lambda}=\left(\Psi_{\Lambda}, \Psi_{\Lambda}\right)=\left\|\Psi_{\Lambda}\right\|^{2}
$$

where $Z_{\Lambda}$ is a partition function. For partial cases of $F$ we have

$$
\left\langle\hat{q}_{[A]} \phi_{\left[A^{\prime}\right]}\left(S_{\Lambda}^{3}\right)\right\rangle_{\Lambda}=Z_{\Lambda}^{-1} \int q_{[A]}\left(\Psi_{\Lambda}\left(q_{\Lambda}\right), \phi_{\left[A^{\prime}\right]}\left(S_{\Lambda}^{3}\right) \Psi_{\Lambda}\left(q_{\Lambda}\right)\right)_{0} d q_{\Lambda},
$$

where the integration is performed over $\mathbb{R}^{|\Lambda|}$ and $\hat{q}_{[A]}$ is the operator of multiplication by $q_{x}$.

We will employ the orthonormal basis $\psi_{\Lambda}^{0}\left(s_{\Lambda}\right)$ of the Euclidean space $\left(\otimes \mathbb{E}^{2}\right)^{|\Lambda|}$, diagonalizing $S_{\Lambda}^{3}$, which is chosen in the following way: $\psi_{\Lambda}^{0}\left(s_{\Lambda}\right)=\otimes_{x \in \Lambda} \psi^{0}\left(s_{x}\right), s_{x}= \pm 1, \psi^{0}(1)=(1,0)$, $\psi^{0}(-1)=(0,1), S^{1} \psi^{0}(s)=\psi^{0}(-s), S^{3} \psi^{0}(s)=s \psi^{0}(s)$. For $F \in \mathbb{L}_{\Lambda}^{2}$ we have the following decomposition

$$
F\left(q_{\Lambda}\right)=\sum_{s_{\Lambda}} F\left(q_{\Lambda} ; s_{\Lambda}\right) \psi_{\Lambda}^{0}\left(s_{\Lambda}\right)
$$

where the summation is performed over the $|\Lambda|$-fold Cartesian product $(-1,1)^{|\Lambda|}$ of the set $(-1,1)$.

The Hamiltonians in (1.1) are employed in hydrogen-bond ferroelectric crystal models, considered in $[1,2,3]$, and describe interaction between heavy ions (oscillators with constant frequency) and protons (spins). The second term with $J_{A}=0,|A| \geq 2$ corresponds to the energy of protons, tunneling along hydrogen bonds from one well to another, and $J_{x}$ is associated with the tunneling frequency. The last term in the expression for our Hamiltonian $V_{A}=\sum_{A \subseteq \Lambda} J_{1}(A) S_{[A]}^{3}$ describes many-body interaction between protons $\left(J_{1}(A)\right.$ is the intensity of the $|A|$-body interaction).

A rigorous analysis of a mean-field version of the Hamiltonian in (1.1) with $\phi_{x}\left(S_{\Lambda}^{3}\right)$ given in (1.2) that is linear in $S^{3}, J_{x} \neq 0, V_{\Lambda}=-(\mu \eta)^{2} \sum_{x \in \Lambda} \phi_{x}^{2}\left(S_{\Lambda}^{3}\right), J_{A}=0$ for $|A| \geq 2$ and $J_{0}(x ; y)$ uniformly in lattice sites proportional to $|\Lambda|^{-1}$ was carried out in [3] in the framework of the Bogolyubov approximating Hamiltonian method [4] and occurrence of spin and oscillator orderings (the corresponding order parameters are non-zero) for two-body interaction between protons at non-zero temperatures was proved. To establish such orderings for ground states without the mean-field limit in a general case is an important task for a theory.

The oscillator and spin orderings are established if one proves the existence of ferromagnetic oscillator and spin long-range orders (lro's) in ground states for the corresponding Hamiltonians. This means that the ground state averages $\left\langle\hat{q}_{x} \hat{q}_{y}\right\rangle_{\Lambda},\left\langle S_{x}^{j} S_{y}^{j}\right\rangle_{\Lambda}, j=1,3$ are bounded uniformly in $\Lambda$ from below by positive numbers. Occurrence of the ferromagnetic lro's implies the existence of the spin order parameters (magnetizations in the first and third directions) $M_{\Lambda}^{l}=|\Lambda|^{-1} \sum_{x \in \Lambda} S_{x}^{l}$, $l=1,3$, and the oscillator order parameter $Q_{\Lambda}=|\Lambda|^{-1} \sum_{x \in \Lambda} q_{x}$ in the thermodynamic limit $\left(\Lambda \rightarrow \mathbb{Z}^{d}\right)$ since the ground state averages of their squares are uniformly bounded in $\Lambda$ from below by a positive number. 
In this paper we find functions $V_{\Lambda}$, depending on oscillator variables, for which ground states or eigenstates $\Psi_{\Lambda}$ of the Hamiltonians in (1.1) are Gibbsian

$$
\Psi_{\Lambda}\left(q_{\Lambda}\right)=\sum_{s_{\Lambda}} e^{-\frac{1}{2} U\left(s_{\Lambda} ; q_{\Lambda}\right)} \psi_{\Lambda}^{0}\left(s_{\Lambda}\right) \psi_{0 \Lambda}\left(q_{\Lambda}\right),
$$

with the linear in $q_{\Lambda}$ spin-oscillator quasi-potential energy $U$

$$
U\left(s_{\Lambda} ; q_{\Lambda}\right)=2 \mu \eta \sum_{x \in \Lambda} q_{x} \phi_{x}\left(s_{\Lambda}\right)+U^{0}\left(s_{\Lambda}\right),
$$

where $\psi_{0 \Lambda}\left(q_{\Lambda}\right)=\prod_{x \in \Lambda} \psi_{0}\left(q_{x}\right), \psi_{0}(q)=\left(\mu \pi^{-1}\right)^{\frac{1}{4}} \exp \left\{-\frac{\mu}{2} q^{2}\right\}$, is the ground state of the free oscillator Hamiltonian, that is the first term in the right-hand side of (1.1) with $\eta=0$. We prove the maximal ordering in the corresponding systems (provided some simple conditions on $U^{0}$ and $\phi_{x}$ are satisfied): magnetizations $M_{\Lambda}^{l}, l=1,3, Q_{\Lambda}$ are non-zero. No other ground states are known with such the property.

Gibbsian ground states were introduced by Kirkwood and Thomas in [5] in XZ spin- $\frac{1}{2}$ models with Hamiltonians (linear in $S^{1}$ ) that include the spin part of (1.1), i.e. the second and third terms, with only $J_{x} \neq 0$ and the periodic boundary condition (this boundary condition is not essential). Matsui in [6,7] enlarged a class of spin- $\frac{1}{2} X Z$-type models in which Gibbsian states exist. The method was further developed by Datta and Kennedy in [8]. An application of classical spins systems for constructing of quantum states was given in [9].

In [10] we showed how to find $V_{\Lambda}$ for a given Gibbsian ground state and established existence of lro's in $S^{1}$ and $S^{3}$ for a wide class of the spin- $\frac{1}{2} \mathrm{XZ}$ models (see also [11, 12]). This reference contains the most simple proofs of the existence of lro's in ground states of quantum many-body systems. A reader may find a review of the results concerning several quantum orders in it (see also $[13,14,15])$.

The ground state in (1.3) can be represented in the following equivalent form

$$
\Psi_{\Lambda}\left(q_{\Lambda}\right)=\sum_{s_{\Lambda}} e^{-\frac{1}{2} U_{*}\left(s_{\Lambda}\right)} \psi_{\Lambda}^{0}\left(s_{\Lambda}\right) \psi_{0 \Lambda}\left(q_{\Lambda}+\eta \phi_{\Lambda}\left(s_{\Lambda}\right)\right), \quad U_{*}=U^{0}-\mu \eta^{2} \sum_{x \in \Lambda} \phi_{x}^{2} .
$$

From (1.5), orthonormality of the basis (see the beginning of the third section) and the equalities $\int \psi_{0}^{2}(q) d q=1, \int \psi_{0}^{2}(q) q d q=0$ it follows that

$$
\left\langle\hat{q}_{x} \hat{q}_{y}\right\rangle_{\Lambda}=\eta^{2}\left\langle\phi_{x}\left(S_{\Lambda}^{3}\right) \phi_{y}\left(S_{\Lambda}^{3}\right)\right\rangle_{\Lambda}=\eta^{2}\left\langle\phi_{x}\left(\sigma_{\Lambda}\right) \phi_{y}\left(\sigma_{\Lambda}\right)\right\rangle_{* \Lambda}, \quad\left\langle S_{x}^{3} S_{y}^{3}\right\rangle_{\Lambda}=\left\langle\sigma_{x} \sigma_{y}\right\rangle_{* \Lambda},
$$

where $\sigma_{x}\left(s_{\Lambda}\right)=s_{x}$ and

$$
\left\langle\phi_{x}\left(\sigma_{\Lambda}\right) \phi_{y}\left(\sigma_{\Lambda}\right)\right\rangle_{* \Lambda}=Z_{* \Lambda}^{-1} \sum_{s_{\Lambda}} e^{-U_{*}\left(s_{\Lambda}\right)} \phi_{x}\left(s_{\Lambda}\right) \phi_{y}\left(s_{\Lambda}\right), \quad Z_{* \Lambda}=\sum_{s_{\Lambda}} e^{-U_{*}\left(s_{\Lambda}\right)} .
$$

Equalities in (1.6) reduce a calculation of averages in our quantum systems to a calculation of averages indexed by a star in Ising models. For a short-range ferromagnetic potential energy $U_{*}$ the first Griffiths inequality holds: $\left\langle\sigma_{[A]}\right\rangle_{* \Lambda} \geq 0$. As a result the following statement (principle) is true.

Proposition 1. Let $J_{0} \geq 0$ in (1.2) and ferromagnetic lro occur in the Ising model with the ferromagnetic potential energy $U_{*}$ given by (1.5), that is $\left\langle\sigma_{x} \sigma_{y}\right\rangle_{* \Lambda}>0$ uniformly in $\Lambda$, then ferromagnetic lro occurs in oscillator variables and $S^{3}$ in the quantum spin-oscillator system with the Hamiltonian (1.1) and ground state $\Psi_{\Lambda}$ in (1.3).

Usefulness of Gibbsian ground states is explained by comparative simplicity of a proof of existence of lro. Our results show that Gibbsian ground states are expected to appear in many quantum spin-oscillator systems with non-trivial interactions.

Our paper is organized as follows. In Section 2 we formulate our main results in a lemma and theorem. In next sections we prove them. 


\section{Main result}

We establish that Gibbsian ground states exist for $J_{A}$ depending on $q_{\Lambda}$ if

$$
V_{\Lambda}=-\sum_{A \subseteq \Lambda} J_{A} e^{-\frac{1}{2} W_{A}\left(S_{\Lambda}^{3}\right)}, \quad W_{A}\left(S_{\Lambda}^{3}\right)=U\left(S_{\Lambda}^{3 A} ; q_{\Lambda}\right)-U\left(S_{\Lambda}^{3} ; q_{\Lambda}\right)
$$

where $S_{\Lambda}^{3 A}=\left(-S_{A}^{3}, S_{\Lambda \backslash A}^{3}\right)$. $V_{\Lambda}$ determines an unbounded operator. Negative $J_{A}$ generate positive functions $V_{\Lambda}$ and this enables us to prove the following lemma.

Lemma 1. Let $V_{\Lambda}$ be given by (2.1) and have a domain $D\left(V_{\Lambda}\right), J_{A}$ be bounded negative functions and $U$ in (2.1) coincide with $U$ in (1.4) Then $H_{\Lambda}$ is positive definite and essentially self-adjoint on the set $\left(\otimes \mathbb{C}^{2}\right)^{|\Lambda|} \otimes \mathbb{S}\left(\mathbb{R}^{|\Lambda|}\right) \cap D\left(V_{\Lambda}\right)$ that contains $\left(\otimes \mathbb{C}^{2}\right)^{|\Lambda|} \otimes C_{0}^{\infty}\left(\mathbb{R}^{|\Lambda|}\right)$ and $\Psi_{\Lambda}$, given by $(1.3)$, is an eigenfunction of the Hamiltonian (1.1) with the zero eigenvalue and is its (unique) ground state if $J_{A} \leq 0$ (if the uniform bound $J_{x} \leq J_{-}<0$ holds).

Remark 1. If the functions $J_{A}$ are only negative then $\Psi_{\Lambda}$ is the ground state of the self-adjoint extension of the Hamiltonian preserving positive definiteness.

The most simple translation invariant short-range $U^{0}$ is ferromagnetic

$$
U^{0}\left(s_{\Lambda}\right)=-\alpha \sum_{A \subseteq \Lambda} J_{A}^{0} s_{[A]}, \quad J_{A}^{0} \geq 0
$$

where $J_{A}^{0}=0$ for odd $|A|, \alpha \geq 0$.

Theorem 1. Let all the conditions of Lemma 1 be satisfied, $J_{0} \geq 0, J_{0}(x ; A)=0$ for even $|A|$, $J_{0}(0 ; 1), J_{0,1}^{0} \geq \bar{J}$ and $U^{0}$ be given by $(2.2)$. Then

I. For a sufficiently large $\beta=\left(\eta^{2} \mu+\alpha\right) \bar{J}>1$ there exist ground state lro's in $S^{3}$ and oscillator variables for $d \geq 2$;

II. Let the positive constants $C, B_{j}, j=1,2$, independent of $\Lambda$, exist such that $\left|\phi_{x}\left(s_{\Lambda}\right)\right| \leq C$

$$
W_{A}^{(j)}\left(s_{\Lambda}\right)=\sum_{x^{\prime} \in \Lambda}\left|\phi_{x^{\prime}}^{j}\left(s_{\Lambda}^{A}\right)-\phi_{x^{\prime}}^{j}\left(s_{\Lambda}\right)\right| \leq B_{j}, \quad j=1,2
$$

where $|A|=2$. Then ground state ferromagnetic lro in $S^{1}$ occurs in arbitrary dimension d.

If $U^{0}=0$ then $V_{\Lambda}$ from $(2.1)$ is given by

$$
V_{\Lambda}=-\sum_{A \subseteq \Lambda} J_{A} v_{[A]}, \quad v_{x}=\cosh u_{x}+S_{x}^{3} \sinh u_{x}, \quad u_{x}=2 \eta \mu \phi_{x}\left(q_{\Lambda}\right)
$$

This equality follows from the equalities

$$
W_{A}\left(S_{\Lambda}^{3}\right)=-2 \sum_{x \in A} u_{x} S_{x}^{3}, \quad e^{a S^{3}}=\cosh a+S^{3} \sinh a, \quad\left(S^{3}\right)^{2}=I .
$$

In the simplest case the conditions of item II of Theorem 1 can be checked without difficulty.

Proposition 2. Let $\phi_{x}$ be linear in $S^{3}$ in (1.2) and $\left\|J_{0}\right\|_{1}=\sum_{x}\left|J_{0}(x)\right|<\infty$, where the summation is performed over $\mathbb{Z}^{d}$. Then the conditions of item II of Theorem 1 are satisfied. 
Note that if one uses the Pauli matrices with $\frac{1}{2}$ instead of the unity as matrix elements then $V_{\Lambda}$ should be changed by adding to $W_{A}$ the number $-|A| \ln 2$.

If one chooses the anti-ferromagnetic $U^{0}$ in (2.1), specifically,

$$
U^{0}\left(s_{\Lambda}\right)=\mu \eta^{2} \sum_{x \in \Lambda} \phi_{x}^{2}\left(s_{\Lambda}\right)+\alpha \sum_{\langle x, y\rangle \in \Lambda} s_{x} s_{y}, \quad \alpha>0
$$

where $x, y$ are nearest neighbors, then it can be easily proved that the spin lro in the third direction will be anti-ferromagnetic, generating a staggered magnetization (spins at the even and odd sublattices take different values).

The interesting and important property of the Hamiltonians with $V_{\Lambda}$ given by $(2.1)$ is that they are simply related to generators of stationary Markovian processes (see, also, [16]). We believe that it is possible to apply the same mathematical technique for proving existence of order and phase transitions in equilibrium quantum systems and non-equilibrium stochastic systems (see [17]).

\section{Proof of Lemma 1}

For our purpose it is convenient to pass to a new representation. It is determined by the Hilbert space of sequences of functions $F\left(q_{\Lambda} ; s_{\Lambda}\right), s_{x}= \pm 1$, which are found in the expansion of the vector $F \in \mathbb{L}_{\Lambda}^{2}$ mentioned at the beginning of the introduction, with the scalar product

$$
\begin{aligned}
& \left(F_{1}, F_{2}\right)=\sum_{s_{\Lambda}} \int F_{1}\left(q_{\Lambda} ; s_{\Lambda}\right) F_{2}\left(q_{\Lambda} ; s_{\Lambda}\right) d q_{\Lambda}, \\
& \left(F_{1}\left(q_{\Lambda}\right), F_{2}\left(q_{\Lambda}\right)\right)_{0}=\sum_{s_{\Lambda}} F_{1}\left(q_{\Lambda} ; s_{\Lambda}\right) F_{2}\left(q_{\Lambda} ; s_{\Lambda}\right),
\end{aligned}
$$

where the integration is performed over $\mathbb{R}^{|\Lambda|}$. Here we took into account the orthonormality of the basis, i.e. the equality

$$
\left(\Psi_{\Lambda}^{0}\left(s_{\Lambda}\right), \Psi_{\Lambda}^{0}\left(s_{\Lambda}^{\prime}\right)\right)_{0}=\delta\left(s_{\Lambda} ; s_{\Lambda}^{\prime}\right)=\prod_{x \in \Lambda} \delta_{s_{x}, s_{x}^{\prime}},
$$

where $\delta_{s, s^{\prime}}$ is the Kronecker delta. Let

$$
\begin{aligned}
& h_{x}=-\partial_{x}^{2}+\mu^{2}\left(q_{x}+\eta \phi_{x}\left(S_{\Lambda}^{3}\right)\right)^{2}-\mu, \\
& h_{x}^{0}=-\partial_{x}^{2}+\mu^{2} q_{x}^{2}-\mu=\left(-\partial_{x}+\mu q_{x}\right)\left(\partial_{x}+\mu q_{x}\right)
\end{aligned}
$$

and

$$
\begin{aligned}
& h_{\Lambda}=\sum_{x \in \Lambda} h_{x}, \quad h_{\Lambda}^{0}=\sum_{x \in \Lambda} h_{x}^{0} \\
& \left(T_{x}(\phi) F\right)\left(q_{\Lambda}\right)=\sum_{s_{\Lambda}} F\left(q_{x}+\eta \phi_{x}\left(s_{\Lambda}\right), q_{\Lambda \backslash x} ; s_{\Lambda}\right) \psi_{\Lambda}^{0}\left(s_{\Lambda}\right),
\end{aligned}
$$

then

$$
h_{x}=T_{x}(\phi) h_{x}^{0} T_{x}^{-1}(\phi), \quad h_{\Lambda}=T_{\Lambda} h_{\Lambda}^{0} T_{\Lambda}^{-1} .
$$

Here we took into account that differentiation commutes with $T_{\Lambda}$.

Our Hamiltonian is rewritten as follows

$$
H_{\Lambda}=h_{\Lambda}+\sum_{A \subseteq \Lambda} J_{A} P_{[A]}, \quad P_{A}=S_{[A]}^{1}-e^{-\frac{1}{2} W_{A}\left(S_{\Lambda}^{3}\right)} .
$$


The remarkable fact is that the symmetric operator $P_{A}$ and the harmonic operator $h_{x}$ have both common eigenvector $\Psi_{\Lambda}$ with the zero eigenvalue (see Remark 3 in the end of the paper). Note that the space of ground states of the operator $h_{x}$ (eigenfunctions with the zero eigenvalue) is $2^{|\Lambda|}$-fold degenerate since $S_{x}^{3}$ is diagonal and the Laplacian is translation invariant. From (1.4) and the definition of $T_{x}(\phi)$ if follows that $T_{x}^{-1}(\phi) \Psi_{\Lambda}$ is equal to $\psi_{0}\left(q_{x}\right)$ multiplied by a function independent of $q_{x}$

$$
T_{x}^{-1}(\phi) \Psi_{\Lambda}=\sum_{s_{\Lambda}} e^{-\frac{1}{2} U_{*}\left(s_{\Lambda}\right)} \psi_{\Lambda}^{0}\left(s_{\Lambda}\right) \psi_{0 \Lambda}\left(q_{\Lambda \backslash x}+\eta \phi_{\Lambda \backslash x}\left(s_{\Lambda \backslash x}\right)\right) \psi_{0}\left(q_{x}\right) .
$$

Hence $h_{x}^{0} T_{x}^{-1}(\phi) \Psi_{\Lambda}=0$ and $h_{x} \Psi_{\Lambda}=T_{x}(\phi) h_{x}^{0} T_{x}^{-1}(\phi) \Psi_{\Lambda}=0$. The proof that $\Psi_{\Lambda}$ is an eigenvector with the zero eigenvalue of $P_{A}$ is inspired by our previous paper [10]. For simplicity we will omit $q_{\Lambda}$ in the expression for $U$ in (1.3). Taking into consideration the equalities

$$
S_{[A]}^{1} \psi_{\Lambda}^{0}\left(s_{\Lambda}\right)=\psi_{\Lambda}^{0}\left(s_{\Lambda}^{A}\right)=\psi_{\Lambda}^{0}\left(s_{\Lambda \backslash A},-s_{A}\right), \quad S_{x}^{3} \psi_{\Lambda}^{0}\left(s_{\Lambda}\right)=s_{x} \psi_{\Lambda}^{0}\left(s_{\Lambda}\right),
$$

we obtain

$$
\begin{aligned}
\left(\psi_{0 \Lambda}\right)^{-1} P_{A} \Psi_{\Lambda} & =\sum_{s_{\Lambda}}\left(\psi_{\Lambda}^{0}\left(s_{\Lambda \backslash A},-s_{A}\right)-e^{-\frac{1}{2} W_{A}\left(s_{\Lambda}\right)} \psi_{\Lambda}^{0}\left(s_{\Lambda}\right)\right) e^{-\frac{1}{2} U\left(s_{\Lambda}\right)} \\
& =\sum_{s_{\Lambda}}\left(\psi_{\Lambda}^{0}\left(s_{\Lambda \backslash A},-s_{A}\right) e^{-\frac{1}{2} U\left(s_{\Lambda}\right)}-\psi_{\Lambda}^{0}\left(s_{\Lambda}\right) e^{-\frac{1}{2} U\left(s_{\Lambda}^{A}\right)}\right) \\
& =\sum_{s_{\Lambda}}\left(e^{-\frac{1}{2} U\left(s_{\Lambda}^{A}\right)}-e^{-\frac{1}{2} U\left(s_{\Lambda}^{A}\right)}\right) \psi_{\Lambda}^{0}\left(s_{\Lambda}\right)=0 .
\end{aligned}
$$

Here we changed signs of the spin variables $s_{A}$ in the first term in the sum in $s_{\Lambda}$.

Positive definiteness of the Hamiltonian follows from the following proposition.

Proposition 3. The operator $-P_{A}$ is positive definite on $D\left(V_{\Lambda}\right)$.

Proof. $V_{\Lambda}$ is an operator of multiplication by infinite differentiable functions on each onedimensional spin subspace and its domain contains $\left(\otimes \mathbb{C}^{2}\right)^{|\Lambda|} \otimes C_{0}^{\infty}\left(\mathbb{R}^{|\Lambda|}\right)$. This domain coincides with the direct sum of $2^{|\Lambda|}$ copies of of $L^{2}\left(\mathbb{R}^{|\Lambda|}, e^{|q|_{0}} d q_{\Lambda}\right)$, where $|q|_{0}=\sum_{x \in \Lambda}\left|q_{x}\right|\left|\phi_{x}\right|$.

The scalar product in the Hilbert space $\mathbb{L}_{\Lambda}^{2}$ is given by $\left(F_{1}, F_{2}\right)=\int\left(F_{1}\left(q_{\Lambda}\right), F_{2}\left(q_{\Lambda}\right)\right) d q_{\Lambda}$, where the integration is performed over $\mathbb{R}^{|\Lambda|}$. We have to show that $-\left(P_{A} F\left(q_{\Lambda}\right), F\left(q_{\Lambda}\right)\right)_{0} \geq 0$. Let us define the operator

$$
P_{A}^{+}=e^{\frac{1}{2} U\left(S_{\Lambda}^{3}\right)} P_{A} e^{-\frac{1}{2} U\left(S_{\Lambda}^{3}\right)} .
$$

It is not difficult to check on the basis $\psi_{\Lambda}^{0}$ that

$$
P_{A}^{+}=e^{-\frac{1}{2} W_{A}\left(S_{\Lambda}^{3}\right)}\left(S_{[A]}^{1}-I\right),
$$

where $I$ is the unit operator. Here we used the following equality

$$
e^{-\frac{1}{2} U\left(S_{\Lambda}^{3 A}\right)} S_{[A]}^{1}=S_{[A]}^{1} e^{-\frac{1}{2} U\left(S_{\Lambda}^{3}\right)} .
$$

For the operator $P_{A}^{+}$we have

$$
P_{A}^{+} F=\sum_{s_{\Lambda}}\left(P_{A}^{+} F\right)\left(q_{\Lambda} ; s_{\Lambda}\right) \psi_{\Lambda}^{0}\left(s_{\Lambda}\right)
$$

and

$$
\left(P_{A}^{+} F\right)\left(q_{\Lambda} ; s_{\Lambda}\right)=-e^{-\frac{1}{2} W_{A}\left(s_{\Lambda}\right)}\left(F\left(q_{\Lambda} ; s_{\Lambda}\right)-F\left(q_{\Lambda} ; s_{\Lambda}^{A}\right)\right) .
$$


It is convenient to introduce the new scalar product

$$
\begin{aligned}
\left(F_{1}, F_{2}\right)_{U} & =\left(e^{-U\left(S_{\Lambda}^{3}\right)} F_{1}, F_{2}\right)=\sum_{s_{\Lambda}} \int F_{1}\left(q_{\Lambda}, s_{\Lambda}\right) F_{2}\left(q_{\Lambda}, s_{\Lambda}\right) e^{-U\left(s_{\Lambda}\right)} d q_{\Lambda} \\
& =\int\left(F_{1}\left(q_{\Lambda}\right), F_{2}\left(q_{\Lambda}\right)\right)_{U}^{0} d q_{\Lambda}=\int\left(e^{-U\left(S_{\Lambda}^{3}\right)} F_{1}\left(q_{\Lambda}\right), F_{2}\left(q_{\Lambda}\right)\right)_{0} d q_{\Lambda} .
\end{aligned}
$$

The operator $P_{A}^{+}$is symmetric with respect to the new scalar product since

$$
\left(P_{A}^{+} F_{1}\left(q_{\Lambda}\right), F_{2}\left(q_{\Lambda}\right)\right)_{U}^{0}=\left(P_{A} e^{-\frac{1}{2} U\left(S_{\Lambda}^{3}\right)} F_{1}\left(q_{\Lambda}\right), e^{-\frac{1}{2} U\left(S_{\Lambda}^{3}\right)} F_{2}\left(q_{\Lambda}\right)\right)_{0}
$$

It is not difficult to check that

$$
\begin{aligned}
-\left(P_{A}^{+} F\left(q_{\Lambda}\right), F\left(q_{\Lambda}\right)\right)_{U}^{0} & =\sum_{s_{\Lambda}} e^{-\frac{1}{2}\left[U\left(s_{\Lambda}\right)+U\left(s_{\Lambda}^{A}\right)\right]}\left(F\left(q_{\Lambda} ; s_{\Lambda}\right)-F\left(q_{\Lambda} ; s_{\Lambda}^{A}\right)\right) F\left(q_{\Lambda} ; s_{\Lambda}\right) \\
& =\frac{1}{2} \sum_{s_{\Lambda}} e^{-\frac{1}{2}\left[U\left(s_{\Lambda}\right)+U\left(s_{\Lambda}^{A}\right)\right]}\left(F\left(q_{\Lambda} ; s_{\Lambda}\right)-F\left(q_{\Lambda} ; s_{\Lambda}^{A}\right)\right)^{2} \geq 0 .
\end{aligned}
$$

Here we took into account that the function under the sign of exponent is invariant under changing signs of the spin variables $s_{A}$. From (3.1), (3.2) it follows that $-P_{A} \geq 0$.

From Proposition 3 it follows that $H_{\Lambda}^{1}=\sum_{A \subseteq \Lambda} J_{A} P_{A}$ is positive definite since for the negative $J_{A}$ we have

$$
\left(H_{\Lambda}^{1} F, F\right)=\sum_{A \subseteq \Lambda} \int J_{A}\left(q_{\Lambda}\right)\left(P_{A} F\left(q_{\Lambda}\right), F\left(q_{\Lambda}\right)\right)_{0} d q_{\Lambda} \geq 0 .
$$

The fact that the Hamiltonian is essentially self-adjoint is derived from the following proposition (see example X.9.3 in [18]).

Proposition 4. The operator $-\Delta+\mu^{2} x^{2}+V+(y, x)$, where $(y, x)$ is the Euclidean scalar product of $x=x_{j}, j=1, \ldots, n$ with the constant vector $y, \Delta=\sum_{j=1}^{n} \frac{\partial}{\partial x_{j}}, \mu \neq 0$ if $y \neq 0, V$ is the operator of multiplication by a non-negative function $V(x) \in L^{2}\left(\mathbb{R}^{n}, e^{-x^{2}} d x\right), x^{2}=(x, x)$, is essentially self-adjoint on $\mathbb{S}\left(\mathbb{R}^{n}\right) \cap D(V)$, i.e. the intersection of the Schwartz space and the domain of $V$.

Proof. The following two inequalities are valid

$$
\mu^{2}\left\|x^{2} \psi\right\| \leq\left\|\left(-\Delta+V+\mu^{2} x^{2}\right) \psi\right\|+2 \mu^{2} n\|\psi\|, \quad(y, x) \leq \epsilon x^{2}+\frac{n}{4 \epsilon}|y|^{2},
$$

where $\mu$ is a real number, $|y|=\max _{j}|y|_{j},\|\cdot\|$ is the scalar product in the Hilbert space of square integrable functions. We tacitly assume that $x^{2}$ means the operator of multiplication of a squared variable. The first inequality follows from the inequalities $\left(\partial_{j}=\frac{\partial}{\partial x_{j}}\right)$

$$
\begin{aligned}
& \left(-\Delta+V+\mu^{2} x^{2}\right)^{2} \geq\left(\mu^{2} x^{2}\right)^{2}-\mu^{2}\left(\Delta x^{2}+x^{2} \Delta\right), \\
& -\left(\partial_{j}^{2} x_{k}^{2}+x_{k}^{2} \partial_{j}^{2}\right)=-\left(2 x_{k} \partial_{j}^{2} x_{k}+2 \delta_{j, k}\right) \geq-2 \delta_{j, k}, \\
& -\left(\Delta x^{2}+x^{2} \Delta\right) \geq-\sum_{j=1}^{n}\left(\partial_{j}^{2} x_{j}^{2}+x_{j}^{2} \partial_{j}^{2}\right) .
\end{aligned}
$$


Here we took into account that $V$ is positive and $\left[\partial_{j}, x_{k}\right]=\partial_{j} x_{k}-x_{k} \partial_{j}=\delta_{j, k}$. As a result

$$
\|(y, x) \psi\| \leq a\left\|\left(-\Delta+V+\mu^{2} x^{2}\right) \psi\right\|+b\|\psi\|, \quad a=\mu^{-2} \epsilon, \quad b=n\left(2+\frac{|y|^{2}}{4 \epsilon}\right) .
$$

For $\mu \neq 0$ number $a$ can be arbitrary small and from the Kato-Rellich theorem [18] it follows that the essential domain of $-\Delta+V+\mu^{2} x^{2}+(y, x)$ coincides with the essential domain of $-\Delta+V+\mu^{2} x^{2}$. But the last one coincides with $\mathbb{S}\left(\mathbb{R}^{n}\right) \cap D(V)$ [18, Theorem X.59]. Inequality (3.3) is sufficient, also, for the proof of the proposition in the case $\mu=y=0$ (this is explained in Example X.3 in [18]).

Since $S_{\Lambda}^{3}$ is a diagonal operator on $\left(\otimes \mathbb{C}^{2}\right)^{|\Lambda|}$ the operator

$$
-\sum_{x \in \Lambda} \partial_{x}^{2}+\mu^{2} \sum_{x \in \Lambda} q_{x}^{2}+V_{\Lambda}+2 \eta \mu^{2} \sum_{x \in \Lambda} q_{x} \phi_{x}\left(S_{\Lambda}^{3}\right)
$$

is the direct sum of $2^{|\Lambda|}$ copies of the minus $|\Lambda|$-dimensional Laplacian plus the three functions coinciding with the three functions in Proposition 4. From Proposition 4 it follows that this operator is essentially self-adjoint on the set $\left(\otimes \mathbb{C}^{2}\right)^{|\Lambda|} \otimes \mathbb{S}\left(\mathbb{R}^{|\Lambda|}\right) \cap D\left(V_{\Lambda}\right)$ that contains $\left(\otimes \mathbb{C}^{2}\right)^{|\Lambda|} \otimes$ $\mathbb{C}_{0}^{\infty}\left(\mathbb{R}^{|\Lambda|}\right)$. The same is true for the Hamiltonian since operator $(\eta \mu)^{2} \sum_{x \in \Lambda} \phi_{x}^{2}\left(S_{\Lambda}^{3}\right)$ and the operator depending on $S_{\Lambda}^{1}$ in its expression are bounded.

The operators $h_{\Lambda}, H_{\Lambda}^{1}$ are positive definite on the dense set $\left(\otimes \mathbb{C}^{2}\right)^{|\Lambda|} \otimes \mathbb{S}\left(\mathbb{R}^{|\Lambda|}\right) \cap D\left(V_{\Lambda}\right)$ which is the essential set for $H_{\Lambda}$. This implies that $H_{\Lambda}$ is positive definite on its domain $D\left(H_{\Lambda}\right)$ and $\Psi_{\Lambda}$ is its ground state.

Proof of uniqueness. We have to establish that the symmetric semigroup $P_{\Lambda}^{t}$, generated by $-H_{\Lambda}$, maps non-negative functions into (strictly) positive functions (increases positivity) and this will imply that the ground state is unique (see Theorem XIII.44 in [18]). We will establish this property with the help of a perturbation expansion. The kernel of the semigroup $P_{1}^{t}$, generated by $h_{\Lambda}+V_{\Lambda}$, is expressed in terms of the Feynman-Kac $(\mathrm{FK})$ formula $[18,19]$

$$
P_{1}^{t}\left(q_{\Lambda}, s_{\Lambda} ; q_{\Lambda}^{\prime}, s_{\Lambda}^{\prime}\right)=\delta_{s_{\Lambda}, s_{\Lambda}^{\prime}} \int P_{q_{\Lambda}, q_{\Lambda}^{\prime}}^{t}\left(d w_{\Lambda}\right) \exp \left\{-\int_{0}^{t} V_{\Lambda}^{+}\left(w_{\Lambda}(\tau), s_{\Lambda}\right) d \tau\right\},
$$

where $V_{\Lambda}^{+}\left(q_{\Lambda} ; s_{\Lambda}\right)=V_{\Lambda}\left(q_{\Lambda} ; s_{\Lambda}\right)+\sum_{x \in \Lambda}\left[\mu^{2}\left(q_{x}+\eta \phi_{x}\left(s_{\Lambda}\right)\right)^{2}-\mu\right], P_{q_{\Lambda}, q_{\Lambda}^{\prime}}^{t}\left(d w_{\Lambda}\right)=\prod_{x \in \Lambda} P_{q_{x}, q_{x}^{\prime}}^{t}\left(d w_{x}\right)$ is the conditional Wiener measure and $w_{\Lambda}(t)$ is the sequence of continuous paths. The semigroup $P^{t}$ is represented as a perturbation series in powers of $V_{0}$

$$
V_{0}=-\sum_{A \subseteq \Lambda} J_{A} S_{[A]}^{1}
$$

This series is convergent in the uniform operator norm [20] since $V_{0}$ is a bounded operator. Its perturbation expansion is given by

$$
P^{t}=\sum_{n \geq 0} P_{n}^{t}, \quad P_{n}^{t}=\int_{0 \leq \tau_{1} \leq \tau_{2} \leq \cdots \leq \tau_{n} \leq t} d \tau_{1} \cdots d \tau_{n} P_{1}^{\tau_{1}} \prod_{j=2}^{n+1}\left(V_{0} P_{1}^{\tau_{j}-\tau_{j-1}}\right),
$$

where $\tau_{n+1}=t$. We now use the following simple inequality

$$
\int_{0}^{t}\left|V_{\Lambda}^{+}\left(w_{\Lambda}(\tau), s_{\Lambda}\right)\right| d \tau
$$




$$
\leq \bar{V}\left(w_{\Lambda}\right)=|J|_{\Lambda} \int_{0}^{t}\left[\exp \left\{\alpha \bar{U}_{0}+2 \eta \mu \sum_{x \in \Lambda} \bar{\phi}_{x}\left|w_{x}(\tau)\right|\right\}+\sum_{x \in \Lambda}\left(\mu^{2}\left(\left|w_{x}(\tau)\right|+\eta \bar{\phi}_{x}\right)^{2}-\mu\right)\right] d \tau
$$

where $\bar{U}_{0}=\max _{s_{\Lambda}} U_{0}\left(s_{\Lambda}\right),|J|_{\Lambda}=\sup _{q_{\Lambda}} \sum_{A \subseteq \Lambda}\left|J_{A}\right|, \bar{\phi}_{x}=\max _{s_{\Lambda}}\left|\phi_{x}\left(s_{\Lambda}\right)\right|$. Let

$$
\bar{P}_{1}^{t}\left(q_{\Lambda} ; q_{\Lambda}\right)=\int P_{q_{\Lambda}, q_{\Lambda}^{\prime}}^{t}\left(d w_{\Lambda}\right) e^{-\bar{V}\left(w_{\Lambda}\right)}, \quad V_{-}=-J_{-} \sum_{x \in \Lambda} S_{x}^{1}
$$

then it follows from the positivity of the the kernel $P_{1}^{t}$ and $V_{0}$ that

$$
P^{t}\left(q_{\Lambda}, s_{\Lambda} ; q_{\Lambda}^{\prime}, s_{\Lambda}^{\prime}\right) \geq \bar{P}_{1}^{t}\left(q_{\Lambda} ; q_{\Lambda}\right) \sum_{n \geq 0} \frac{t^{n}}{n !} V_{-}^{n}\left(s_{\Lambda} ; s_{\Lambda}^{\prime}\right)
$$

Here we utilized the semigroup property of $\bar{P}^{t}$, the inequalities $e^{a} \geq e^{-|a|}, V_{0} \geq V_{-}$,

$$
\left(V_{0} P_{1}^{\tau_{j}-\tau_{j-1}}\right)\left(q_{\Lambda}, s_{\Lambda} ; q_{\Lambda}^{\prime}, s_{\Lambda}^{\prime}\right) \geq \bar{P}^{\tau_{j}-\tau_{j-1}}\left(q_{\Lambda} ; q_{\Lambda}^{\prime}\right) V_{-}\left(s_{\Lambda} ;, s_{\Lambda}^{\prime}\right)
$$

Now, it is easily proved as in [10] that the matrix $V_{-}$is irreducible. As a result there exists a positive integer $n$ such that and that $V_{-}^{n}$, has positive non-diagonal elements [21, 22]. Hence the kernel in the left-hand side of (3.4) is positive.

\section{Order parameters}

In this section we will prove Theorem 1, i.e. occurrence of different types of lro in the considered quantum systems. In the proof we will rely on the following basic theorem.

Theorem 2. Let the ferromagnetic short-range potential energy $U$ of the classical Ising model on the hyper-cubic lattice $\mathbb{Z}^{d}$ with the partition function $Z_{\Lambda}=\sum_{s_{\Lambda}} \exp \left\{-\beta U\left(s_{\Lambda}\right)\right\}$ be given by

$$
U\left(s_{\Lambda}\right)=-\sum_{A \subseteq \Lambda} \varphi(A) s_{[A]}
$$

where $\varphi(A) \geq 0, s_{x}= \pm 1$. Let, also, the uniform bound $\varphi(x, y) \geq \bar{\varphi}>0$ for nearest neighbors $x, y$ hold and $\varphi(A)=0$ for $A$ with odd number of sites. Then for a sufficiently large $\bar{\varphi} \beta>1$ and the dimension $d \geq 2$ there is the ferromagnetic lro, that is, for the Gibbsian two point spin average the uniform in $\Lambda$ bound holds

$$
\left\langle\sigma_{x} \sigma_{y}\right\rangle_{\Lambda}>0
$$

where $\sigma_{x}\left(s_{\Lambda}\right)=s_{x}$ and the magnetization (an order parameter) $M_{\Lambda}=|\Lambda|^{-1} \sum_{x \in \Lambda} s_{x}$ is non-zero in the thermodynamic limit $\Lambda \rightarrow \mathbb{Z}^{d}$.

The proof of this theorem is based on an application of the generalized Peierls principle (argument). It will be given in the end of this section (see, also, [17, 26]. The next theorem is the consequence of the basic theorem.

Proof of item I of Theorem 1. Condition (1.2) shows that

$$
\sum_{x \in \Lambda} \phi_{x}^{2}\left(s_{\Lambda}\right)=|\Lambda|+2 \sum_{A \subseteq \Lambda} J_{2}(A) s_{[A]}+\sum_{x \in \Lambda}\left(\sum_{x \notin A \subseteq \Lambda} J_{0}(x ; A) s_{[A]}\right)^{2} .
$$


where $J_{2}(x \cup A)=J_{0}(x ; A)$ and $J_{2}(A)=0$ for odd $|A|$. The last term is equal to

$$
\sum_{x \in \Lambda} \sum_{x \notin A \subseteq \Lambda} J_{0}^{2}(x ; A)+2 \sum_{x \in \Lambda} \sum_{x \notin A_{1}, A_{2} \subseteq \Lambda} J_{0}\left(x ; A_{1}\right) J_{0}\left(x ; A_{2}\right) s_{\left[A_{1} \Delta A_{2}\right]},
$$

where $s_{\varnothing}=1$ and $A_{1} \Delta A_{2}=\left(A_{1} \cup A_{2}\right) \backslash\left(A_{1} \cap A_{2}\right)$. Due to translation invariance of interaction the first term is bounded by $|\Lambda| \sum_{A} J_{0}^{2}(0 ; A)$, where the summation is performed over $\mathbb{Z}^{d}$, and this expression is finite since the interaction is short-range. Hence subtracting a finite constant proportional to $|\Lambda|$ from $U_{*}$ one sees that the result admits representation (4.1) with positive $J_{*}$ instead of $\varphi$ such that $J_{*}(A)=0$ for odd $|A|$ and for nearest neighbors $x, y$ the inequality $J_{*}(x, y) \geq\left(2 \eta^{2} \mu+\alpha\right) \bar{J}$. The basic theorem and Proposition 1 imply occurrence of ferromagnetic lro in $S^{3}$ and oscillator lro.

Proof of item II of Theorem 1. Let $\psi_{\Lambda}\left(q_{\Lambda} ; s_{\Lambda}\right)=\psi_{\Lambda}^{0}\left(s_{\Lambda}\right) \psi_{0 \Lambda}\left(q_{\Lambda}\right)$. Then (1.2) and the definition of $S^{1}$ imply that

$$
S_{x}^{1} S_{y}^{1} \Psi_{\Lambda}\left(q_{\Lambda}\right)=\sum_{s_{\Lambda}} e^{-\frac{1}{2} U\left(s_{\Lambda} ; q_{\Lambda}\right)} \psi_{\Lambda}\left(q_{\Lambda} ; s_{\Lambda}^{x, y}\right)=\sum_{s_{\Lambda}} e^{-\frac{1}{2} U\left(s_{\Lambda}^{x, y} ; q_{\Lambda}\right)} \psi_{\Lambda}\left(q_{\Lambda} ; s_{\Lambda}\right)
$$

Taking into account also the orthonormality of the basis one obtains

$$
\begin{aligned}
\left\langle S_{x}^{1} S_{y}^{1}\right\rangle_{\Lambda} & =Z_{\Lambda}^{-1} \sum_{s_{\Lambda}} \int e^{-\frac{1}{2}\left[U\left(s_{\Lambda}^{x, y} ; q_{\Lambda}\right)+U\left(s_{\Lambda} ; q_{\Lambda}\right)\right]} \psi_{0 \Lambda}^{2}\left(q_{\Lambda}\right) d q_{\Lambda} \\
& =Z_{\Lambda}^{-1} \sum_{s_{\Lambda}} e^{\frac{\eta^{2} \mu}{4} \sum_{x^{\prime} \in \Lambda}\left(\phi_{x^{\prime}}\left(s_{\Lambda}\right)+\phi_{x^{\prime}}\left(s_{\Lambda}^{x, y}\right)\right)^{2}} e^{-\frac{\alpha}{2}\left[U_{0}\left(s_{\Lambda}^{x, y}\right)+U_{0}\left(s_{\Lambda}\right)\right]} \\
& \geq e^{-\frac{\alpha}{2} B_{0}} Z_{\Lambda}^{-1} \sum_{s_{\Lambda}} e^{\frac{\eta^{2} \mu}{4} \sum_{x^{\prime} \in \Lambda}\left(\phi_{x^{\prime}}\left(s_{\Lambda}\right)+\phi_{x^{\prime}}\left(s_{\Lambda}^{x, y}\right)\right)^{2}} e^{-\alpha U_{0}\left(s_{\Lambda}\right)} .
\end{aligned}
$$

We also have

$$
\begin{aligned}
\sum_{x^{\prime} \in \Lambda}( & \left.\phi_{x^{\prime}}\left(s_{\Lambda}\right)+\phi_{x^{\prime}}\left(s_{\Lambda}^{x, y}\right)\right)^{2} \\
& =\sum_{x^{\prime} \in \Lambda}\left[3 \phi_{x^{\prime}}^{2}\left(s_{\Lambda}\right)+\phi_{x^{\prime}}^{2}\left(s_{\Lambda}^{x, y}\right)\right]+2 \sum_{x^{\prime} \in \Lambda} \phi_{x^{\prime}}\left(s_{\Lambda}\right)\left[-\phi_{x^{\prime}}\left(s_{\Lambda}\right)+\phi_{x^{\prime}}\left(s_{\Lambda}^{x, y}\right)\right] \\
& =\sum_{x^{\prime} \in \Lambda} 4 \phi_{x^{\prime}}^{2}\left(s_{\Lambda}\right)+\sum_{x^{\prime} \in \Lambda}\left[-\phi_{x^{\prime}}^{2}\left(s_{\Lambda}\right)+\phi_{x^{\prime}}^{2}\left(s_{\Lambda}^{x, y}\right)\right]+2 \sum_{x^{\prime} \in \Lambda} \phi_{x^{\prime}}\left(s_{\Lambda}\right)\left[-\phi_{x^{\prime}}\left(s_{\Lambda}\right)+\phi_{x^{\prime}}\left(s_{\Lambda}^{x, y}\right)\right] \\
& \geq 4 \sum_{x^{\prime} \in \Lambda} \phi_{x^{\prime}}^{2}\left(s_{\Lambda}\right)-B_{2}-2 C B_{1} .
\end{aligned}
$$

This yields

$$
\begin{aligned}
\left\langle S_{x}^{1} S_{y}^{1}\right\rangle_{\Lambda} & \geq e^{-\frac{\eta^{2} \mu}{4}\left(B_{2}+2 B_{1}\right)} e^{-\frac{\alpha}{2} B_{0}} Z_{\Lambda}^{-1} \sum_{s_{\Lambda}} e^{\eta^{2} \mu \sum_{x^{\prime} \in \Lambda} \phi_{x^{\prime}}^{2}\left(s_{\Lambda}\right)} e^{-\alpha U_{0}\left(s_{\Lambda}\right)} \\
& =e^{-\frac{\eta^{2} \mu}{4}\left(B_{2}+2 C B_{1}\right)} e^{-\frac{\alpha}{2} B_{0}} .
\end{aligned}
$$

Proof of Proposition 2. It is obvious that $\left|\phi_{x}\left(s_{\Lambda}\right)\right| \leq\left\|J_{0}\right\|_{1}$ and that

$$
\left|\phi_{x^{\prime}}\left(s_{\Lambda}^{x, y}\right)-\phi_{x^{\prime}}\left(s_{\Lambda}\right)\right|=\left|-2\left[s_{y} J_{0}\left(y-x^{\prime}\right)+s_{x} J_{0}\left(x-x^{\prime}\right)\right]\right| \leq 2\left[\left|J_{0}\left(y-x^{\prime}\right)\right|+\left|J_{0}\left(x-x^{\prime}\right)\right|\right] .
$$


As a result $W_{x, y}^{(1)}\left(s_{\Lambda}\right) \leq 4\left\|J_{0}\right\|_{1}$. Further

$$
\begin{aligned}
\left|\phi_{x^{\prime}}^{2}\left(s_{\Lambda}^{x, y}\right)-\phi_{x^{\prime}}^{2}\left(s_{\Lambda}\right)\right|=\mid\left[\sum_{z \in \Lambda \backslash(x, y)} J_{0}\left(z-x^{\prime}\right) s_{z}-s_{y} J_{0}\left(y-x^{\prime}\right)-s_{x} J_{0}\left(x-x^{\prime}\right)\right]^{2} \\
\quad-\left[\sum_{z \in \Lambda \backslash(x, y)} J_{0}\left(z-x^{\prime}\right) s_{z}+s_{y} J_{0}\left(y-x^{\prime}\right)+s_{x} J_{0}\left(x-x^{\prime}\right)\right]^{2} \mid \\
\quad=\left|-4 \sum_{z \in \Lambda \backslash(x, y)} J_{0}\left(z-x^{\prime}\right) s_{z}\left(s_{y} J_{0}\left(y-x^{\prime}\right)+s_{x} J_{0}\left(x-x^{\prime}\right)\right)\right| \\
\quad \leq 4 \sum_{z \in \Lambda}\left|J_{0}\left(z-x^{\prime}\right)\right|\left(\left|J_{0}\left(y-x^{\prime}\right)\right|+\left|J_{0}\left(x-x^{\prime}\right)\right|\right) \leq 4|| J_{0} \mid{ }_{1}\left(\left|J_{0}\left(y-x^{\prime}\right)\right|+\left|J_{0}\left(x-x^{\prime}\right)\right|\right) .
\end{aligned}
$$

Hence $W_{x, y}^{(2)}\left(s_{\Lambda}\right) \leq 8\left\|J_{0}\right\|_{1}^{2}$.

Proof of Theorem 2. Let $\chi_{x}^{ \pm}=\frac{1}{2}\left(1 \pm \sigma_{x}\right)$ then one obtains

$$
4\left\langle\chi_{x}^{+} \chi_{y}^{-}\right\rangle_{\Lambda}=1+\left\langle\sigma_{x}\right\rangle_{\Lambda}-\left\langle\sigma_{y}\right\rangle_{\Lambda}-\left\langle\sigma_{x} \sigma_{y}\right\rangle_{\Lambda} .
$$

Since the systems are invariant under the transformation of changing signs of spins the third and the second terms in the right-hand side of last equality are equal to zero and

$$
\left\langle\sigma_{x} \sigma_{y}\right\rangle_{\Lambda}=1-4\left\langle\chi_{x}^{+} \chi_{y}^{-}\right\rangle_{\Lambda}
$$

Hence if

$$
\left\langle\chi_{x}^{+} \chi_{y}^{-}\right\rangle_{\Lambda}<\frac{1}{4}
$$

then the ferromagnetic lro occurs, i.e.

$$
\left\langle\sigma_{x} \sigma_{y}\right\rangle_{\Lambda} \geq a>0
$$

where $a$ is independent of $\Lambda$. If one succeeds in proving that there exists a positive function $E_{0}(\beta)$ and positive constants $a, a^{\prime}$ independent of $\Lambda$ such that

$$
\left\langle\chi_{x}^{+} \chi_{y}^{-}\right\rangle_{\Lambda} \leq a^{\prime} e^{E_{0}(\beta)}
$$

and proves that $E_{0}$ is increasing at infinity then (4.2), (4.3) will hold for a sufficiently large inverse temperature $\beta$. The Peierls principle reduces the derivation of (4.4) to the derivation of the contour bound.

Peierls principle. Let the contour bound hold

$$
\left\langle\prod_{\langle x, y\rangle \in \Gamma} \chi_{x}^{+} \chi_{y}^{-}\right\rangle_{\Lambda} \leq e^{-|\Gamma| E},
$$

where $\langle\cdot\rangle_{\Lambda}$ denotes the Gibbs average for the spin system confined to a compact domain $\Lambda$, $\Gamma$ is a set of the nearest neighbors, adjacent to the (connected) contour, i.e. a boundary of the connected set of unit hypercubes centered at lattice sites. Then (4.4) is valid with $E_{0}=a^{\prime \prime} E$, where $a^{\prime \prime}$ is a positive constant independent of $\Lambda$. 
Proof of contour bound. Bricmont and Fontain derived the contour bound for the spin systems with the potential energy (4.1) with the help of the second Griffiths [23] and Jensen inequalities [24] (see also [25, 26])

$$
\left\langle\sigma_{[A]} \sigma_{[B]}\right\rangle_{\Lambda[\Gamma]}-\left\langle\sigma_{[A]}\right\rangle_{\Lambda[\Gamma]}\left\langle\sigma_{[B]}\right\rangle_{\Lambda[\Gamma]} \geq 0, \quad \int e^{f} d \mu \geq \exp \left\{\int f d \mu\right\}
$$

where $d \mu$ is a probability measure on a measurable space. Their proof starts form the inequality

$$
\chi_{x}^{+} \chi_{y}^{-}=e^{-\frac{\beta}{2} \sigma_{x} \sigma_{y}} e^{\frac{\beta}{2} \sigma_{x} \sigma_{y}} \chi_{x}^{+} \chi_{y}^{-} \leq e^{-\frac{\beta}{2} \sigma_{x} \sigma_{y}} \chi_{x}^{+} \chi_{y}^{-} \leq e^{-\frac{\beta}{2} \sigma_{x} \sigma_{y}} .
$$

As a result $\left(\beta^{\prime}=\bar{\varphi} \beta\right)$

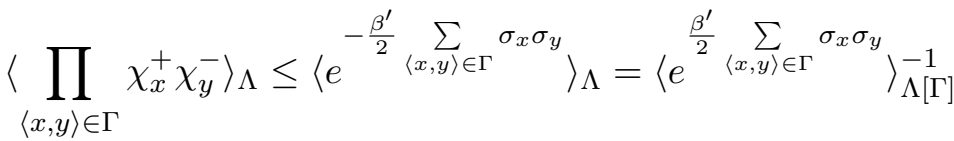

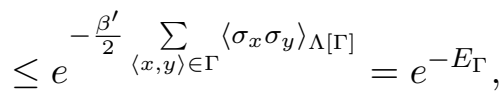

where $\langle\cdot, \cdot\rangle_{\Lambda[\Gamma]}$ is the average corresponding to the potential energy

$$
U_{\Gamma}\left(q_{\Lambda}\right)=U\left(s_{\Lambda}\right)+\frac{\bar{\varphi}}{2} \sum_{\langle x, y\rangle \in \Gamma} s_{x} s_{y} .
$$

In the last line we applied the Jensen inequality. From the second Griffiths inequality it follows that the average $\left\langle\sigma_{x} \sigma_{y}\right\rangle_{\Lambda[\Gamma]}$ is a monotone increasing function in $\varphi_{A}$. So, in the potential energy determining this average we can put $\varphi_{A}=0$, except $A=\langle x, y\rangle$ and leave the coefficient $\bar{\varphi}$ in front of the bilinear nearest-neighbor pair potential in (4.1) without increasing the average. This leads to

$$
\left\langle\sigma_{x} \sigma_{y}\right\rangle_{\Lambda[\Gamma]} \geq\left\langle\sigma \sigma^{\prime}\right\rangle=Z_{2}^{-1}\left(\frac{\beta^{\prime}}{2}\right) \sum_{s_{1}, s_{2}= \pm 1} s_{1} s_{2} e^{\frac{\beta^{\prime}}{2} s_{1} s_{2}}, \quad Z_{2}(\beta)=\sum_{s_{1}, s_{2}= \pm 1} e^{\frac{\beta^{\prime}}{2} s_{1} s_{2}} .
$$

That is,

$$
E_{\Gamma} \geq|\Gamma| E, \quad E=2^{-1} \beta^{\prime}\left\langle\sigma \sigma^{\prime}\right\rangle
$$

or

$$
E=\beta\left(e^{2^{-1} \beta^{\prime}}-e^{-2^{-1} \beta^{\prime}}\right)\left(e^{2^{-1} \beta^{\prime}}+e^{-2^{-1} \beta^{\prime}}\right)^{-1} \geq 2^{-1} \beta^{\prime}\left(1-e^{-\beta^{\prime}}\right) .
$$

Here we used in the denominator the inequality $e^{-2^{-1} \beta^{\prime}} \leq e^{2^{-1} \beta^{\prime}}$. Obviously, $E$ tends to infinity if $\beta^{\prime}$ tends to infinity. This implies (4.5).

\section{Discussion}

We showed that in the considered lattice spin-boson models with $J_{A} \leq 0$ ground states are Gibbsian and the ground state averages for special observables are reduced to averages in classical Ising models. This means that existence of ground states order parameters is connected with existence of order parameters in the associated Ising models and that a breakdown of symmetries in the quantum systems is determined by a breakdown of symmetries in Ising models. We considered the free boundary conditions implying that for the cases of the perturbation $V_{\Lambda}$, 
considered in the two theorems, the ground state averages of $q_{x}, S_{x}^{3}$ are zero, that is $\left\langle\hat{q}_{x}\right\rangle_{\Lambda}=0$, $\left\langle S_{x}^{3}\right\rangle_{\Lambda}=0$ if the associated Ising potential energy is an even function. In order to make such the averages non-zero (explicit symmetry breaking) one has to introduce special boundary conditions (quasi-averages) which have to single out pure Gibbsian states in the associated Ising models. It is known [28] that for the two-dimensional ferromagnetic Ising nearest-neighbor model there are two boundary conditions which generate pure states and that every other state is a convex linear combination of these two states. A discussion of a construction of ground states in lattice spin and fermion quantum systems with an explicit symmetry breaking a reader may find in [29].

Remark 2. Translation invariance means that

$$
J_{x_{1}, \ldots, x_{n}}=J_{0, x_{2}-x_{1}, \ldots, x_{n}-x_{1}}, \quad J_{0}\left(x ; x_{1}, \ldots, x_{n}\right)=J_{0}\left(0 ; x_{1}-x, \ldots, x_{n}-x\right)
$$

where $J, J_{0}$ are symmetric functions. The short-range character of interaction means that

$$
\max _{x} \sum_{A}\left|J_{x, A}\right|<\infty, \quad \max _{x} \sum_{A}\left|J_{0}(x ; A)\right|<\infty .
$$

Remark 3. If only one-point sets are left in the sum for $V_{\Lambda}$ then the expression for $H_{\Lambda}$ can be rewritten in the following way

$$
H_{\Lambda}=\sum_{x \in \Lambda} H_{x}
$$

The property of the ground state $\Psi_{\Lambda}$ to be a ground state with the zero eigenvalue of a local Hamiltonian $H_{x}$ was found earlier for special isotropic anti-ferromagnetic Heisenberg chains with valence bond ground state in [27].

\section{References}

[1] Kobayashi K., Dynamical theory of the phase transition in $\mathrm{KH}_{2} \mathrm{PO}_{4}$-type ferroelectric crystals, J. Phys. Soc. Japan 24 (1968), 497-508.

[2] Villain J., Stamenkovic S., Atomic motion in hydrogen-bond ferroelectrics, Phys. Stat. Sol. 15 (1966), 585596.

[3] Kurbatov A., Plechko V., Exactly solvable model with order-disorder phase transition for ferroelectrics, Teoret. Mat. Fiz. 26 (1976), 109-116.

[4] Bogolyubov N.N. Jr., Brankov J.G., Kurbatov A.M., Tonchev N.C., Zagrebnov V.A., Method of approximating Hamiltonian in statistical physics, Sophia, 1981.

[5] Kirkwood J., Thomas L., Expansions and phase transitions for the ground state of quantum Ising lattice systems, Comm. Math. Phys. 88 (1983), 569-580.

[6] Matsui T., A link between quantum and classical Potts models, J. Statist. Phys. 59 (1990), 781-798.

[7] Matsui T., Uniqueness of the translationally invariant ground state in quantum spin systems, Comm. Math. Phys. 126 (1990), 453-467.

[8] Datta N., Kennedy T., Expansions of one quasiparticle states in spin-1/2 systems, J. Statist. Phys. 108 (2002), 373-399, cond-mat/0104199.

[9] Goderis D., Maes C., Constructing quantum dissipations and their reversible states from classical interacting spin systems, Ann. Inst. H. Poincaré Phys. Théor. 55 (1991), 805-828.

[10] Dorlas T., Skrypnik W., On quantum XZ 1/2 spin models with Gibbsian ground states, J. Phys. A: Math. Gen. 37 (2004), 66623-6632.

[11] Skrypnik W., Order parameters in XXZ-type spin $\frac{1}{2}$ quantum models with Gibbsian ground states, SIGMA 2 (2006), 011, 6 pages, math-ph/0601060.

[12] Skrypnik W., Quantum spin XXZ-type models with Gibbsian ground states and double long-range order, Phys. Lett. A 371 (2007), 363-373. 
[13] Messager A., Nachtergaele B., A model with simultaneous first and second order phase transitions, J. Statist. Phys. 122 (2006), 1-14, cond-mat/0501229.

[14] van Enter A.C.D., Shlosman S.B., First-order transitions for $n$-vector models in two and more dimensions; rigorous proof, Phys. Rev. Lett. 89 (2002), 285702, 3 pages, cond-mat/0205455.

[15] van Enter A.C.D., Shlosman S.B., Provable first-order transitions for liquid crystal and lattice gauge models with continuous symmetries, Comm. Math. Phys. 255 (2005), 21-32, cond-mat/0306362.

[16] Thomas L.E., Quantum Heisenberg ferromagnets and stochastic exclusion processes, J. Math. Phys. 21 (1980), 1921-1924.

[17] Skrypnik W., Long-range order in nonequilibrium systems of interacting Brownian linear oscillators, J. Statist. Phys. 111 (2003), 291-321.

[18] Reed M., Simon B., Methods of modern mathematical physics, Vols. II, IV, Academic Press, 1975.

[19] Ginibre J., Reduced density matrices of quantum gases. I. Limit of infinite volume, J. Math. Phys. 6 (1964), $238-251$.

[20] Kato T., Perturbation theory for linear operators, Springer-Verlag, 1966.

[21] Gantmacher F.R., Matrix theory, 4th ed., Nauka, Moscow, 1988.

[22] Gantmacher F.R., Applications of the theory of matrices, Interscience Publ., New York, 1959.

[23] Kelly D., Sherman S., Inequalities on correlations in Ising ferromagnets, J. Math. Phys. 9 (1968), 466-483.

[24] Bricmont J., Fontaine J.-R., Correlation inequalities and contour estimates, J. Statist. Phys. 26 (1981), $745-753$.

[25] Fröhlich J., Lieb E., Phase transitions in anisotropic lattice spin systems, Comm. Math. Phys. 60 (1978), 233-267.

[26] Skrypnik W., Long-range order in linear ferromagnetic oscillator systems. Strong pair quadratic $n$ - $n$ potential, Ukrainian Math. J. 56 (2004), 964-972.

[27] Affleck I., Kennedy T., Lieb E., Tasaki H., Valence bond ground states in isotropic quantum antiferromagnets, Comm. Math. Phys. 115 (1988), 477-528.

[28] Izenman M., Translation invariance and instability of phase coexistence in the teo dimensional Ising system, Comm. Math. Phys. 73 (1980), 84-92.

[29] Koma T., Tasaki H., Symmetry breaking and finite-size effects in quantum many-body systems, J. Statist. Phys. 76 (1994), 745-803, cond-mat/9708132. 